\title{
On solutions of Fourier's first problem for a system of non-linear parabolic equations in an unbounded domain
}

\author{
by P. Besala (Gdańsk)
}

Introduction. In the papers [5]-[9] M. Krzyżański has proved, among other theorems, some theorems on the existence and uniqueness of the solution of Fourier's first problem (as well as of the Cauchy problem) in an unbounded domain and in the class of functions $E_{2}$ for the linear equation of parabolic type of the normal form (for the definition of class $E_{\delta}$, see $\S 1$ of this paper)

$$
\sum_{i, j=1}^{m} a_{i j}(x, t) \frac{\partial^{2} u}{\partial x_{i} \partial x_{j}}+\sum_{j=1}^{m} b_{j}(x, t) \frac{\partial u}{\partial x_{j}}+c(x, t) u-\frac{\partial u}{\partial t}=f(x, t) .
$$

For this purpose M. Krzyżański has constructed certain auxiliary functions which permit to prove of the above-mentioned theorems for equation (0.1) with unbounded (continuous) coefficients fulfilling the inequalities

$$
\left|a_{i \jmath}(x, t)\right| \leqslant A, \quad\left|b_{j}(x, t)\right| \leqslant A_{1}|x|+B_{1}, \quad c(x, t) \leqslant A_{2}|x|^{2}+B_{2},
$$

$A, A_{1}, A_{2}, B_{1}, B_{2}$ being positive constants, $|x|=\left(\sum_{i=1}^{m} x_{i}^{2}\right)^{1 / 2}$.

In this paper we consider the problem posed by Professor Krzyżański. We deal with a system of non-linear equations with partial derivatives of the parabolic type of the form

$$
\begin{gathered}
\frac{\partial u_{s}}{\partial t}=F_{s}\left(x_{1}, \ldots, x_{m}, t, u_{1}, \ldots, u_{n}, \frac{\partial u_{s}}{\partial x_{1}}, \ldots, \frac{\partial u_{s}}{\partial x_{m}}, \frac{\partial^{2} u_{s}}{\partial x_{1}^{2}}, \frac{\partial^{2} u_{s}}{\partial x_{1} \partial x_{2}}, \ldots, \frac{\partial^{2} u_{s}}{\partial x_{m}^{2}}\right) \\
(s=1, \ldots, n),
\end{gathered}
$$

where in the equation with the index $s$ the derivatives of functions $u_{1}, \ldots, u_{s-1}, u_{s+1}, \ldots, u_{n}$ do not appear.

Theorems I-III dealt with in this paper concern the solutions of Fourier's first boundary-value problem in an unbounded domain (they hold for the Cauchy problem as well). And thus in $\S 3$ we prove the 
theorem on the uniqueness of solutions of this problem in class $E_{2}$. Theorem II in $\$ 4$ concerns certain inequalities between the solutions of two systems of equations of (0.3) form in an unbounded domain and in class $E_{2}$. Theorem III ( $\$ 5$ ) speaks about the existence of a solution of Fourier's first problem in an unbounded domain if we assume the existence of a solution in a bounded domain. We assume the functions appearing in initial and boundary conditions to belong to class $E_{2}$, and we prove that in that case the solution also belongs to class $E_{2}$.

In all the theorems we assume that the functions $F_{s}$ appearing on the right side of the system (0.3) fulfil a certain condition weaker than that of Lipschitz (the so-called (I)-condition; see $\S 2$ ). In the statement on inequalities we assume additionaly that the $F_{\varepsilon}$ functions are not decreasing with regard to some variables.

The method of proving these statements is based on the method applied by $M$. Krzyżański. Using the above-mentioned auxiliary functions constructed by M. Krzyżański we show that the theorems mentioned previously remain true for the (0.3) system embracing equation (0.1) with unbounded coefficients fulfilling the (0.2)-conditions.

Fourier's problems for the system (0.3) have been treated by J. Szarski [17], [18] under weaker assumptions concerning the functions $F_{s}$, but nevertheless in domains whose intersections with hyperplanes $t=$ const are bounded.

A theorem similar to II has been established by W. Mlak [13] in a bounded domain.

The Cauchy problem for parabolic non-linear (quasi-linear) equations has been considered in papers [2], [19].

S. D. Eǐdelman [2] has proved the correctness of this problem (in a sufficiently narrow strip) in class $E_{2}$ for a parabolic system of quasilinear equations and under stronger assumptions than those in this paper, concerning, among other things, the character of increase of the non-linear term together with an increase of $|x|$ and $u_{1}, \ldots, u_{n}$.

In his paper [19] T. D. Ventcel has proved the existence and uniqueness of the solution of the Cauchy problem for a parabolic quasilinear equation under strong assumptions concerning the coefficients and functions appearing in the initial condition.

I wish, here, to express my sincere thanks to Professor M. Krzyżański for posing the problem and for his valuable advice while I was working on that subject. I should also like to thank Professor J. Szarski for his helpful remarks.

§ 1. Notation and definitions. Let us denote by $x\left(x_{1}, x_{2}, \ldots, x_{m}\right)$ a point of the $m$-dimensional Euclidean space $\mathcal{E}^{m}$, and by $\left(x_{1}, x_{2}, \ldots, x_{m}, t\right)$, or shortly $(x, t)$-a point of the space $c^{m+1}$. 
Let $D$ denote an open unbounded domain of $\varepsilon^{m+1}$, lying between the hyperplanes $t=0$ and $t=T>0$, whose boundary $F D$ consists of $m$-dimensional unbounded domains $S^{0}$ and $S^{T}$ of hyperplanes $t=0$, $t=T$ and of surface $\sigma$ which is not tangent to any hyperplane $t=$ const. System (0.3) will be written shortly as

$$
\frac{\partial u_{s}}{\partial t}=F_{s}\left(x, t, u_{i}, \frac{\partial u_{s}}{\partial x_{j}}, \frac{\partial^{2} u_{s}}{\partial x_{j} \partial x_{k}}\right) \quad(s, i=1, \ldots, n ; j, k=1, \ldots, m) .
$$

We assume that the functions $F_{s}\left(x, t, y_{i}, z_{j}, z_{j k}\right)(s=1, \ldots, n)$ are defined in the domain $\Pi$ defined as follows: $(x, t) \in D$ and $y_{i}, z_{j}, z_{j k}$ arbitrary.

Let

$$
w_{1}(x, t), w_{2}(x, t), \ldots, w_{n}(x, t)
$$

be a sequence of functions of class $C^{1}$ in $D$. We will make use of the following definition, due to J. Szarski.

Definition I. An equation with the index 8 of (1.1) is called parabolic with respect to sequence (1.2) if for every system of numbers $z_{j k}, \bar{z}_{j k}$ $(j, k=1, \ldots, m), z_{j k}=z_{k j}, \bar{z}_{j k}=\bar{z}_{k j}$, such that the quadratic form

$$
\sum_{j, k=1}^{m}\left(z_{j k}-\bar{z}_{j k}\right) \lambda_{j} \lambda_{k}
$$

is non-positive for arbitrary vector $\left(\lambda_{1}, \ldots, \lambda_{m}\right)$, the following inequality holds:

$$
\begin{array}{r}
F_{s}\left(x, t, w_{i}(x, t), \frac{\partial w_{s}(x, t)}{\partial x_{j}}, z_{j k}\right)-F_{s}\left(x, t, w_{i}(x, t), \frac{\partial w_{s}(x, t)}{\partial x_{j}}, \bar{z}_{j k}\right) \leqslant 0 \\
\text { for } \quad(x, t) \in D .
\end{array}
$$

If each equation of (1.1) is parabolic with respect to the same sequence, then we say that system (1.1) is parabolic with respect to that sequence. The solution of system (1.1) is called parabolic if this system is parabolic with respect to the solution.

We say that a solution $u_{i}(x, t)(i=1, \ldots, n)$ is regular in $D$ if the functions $u_{i}(x, t)$ are continuous in the closure $\bar{D}$ of $D$ and possess continuous derivatives appearing in the equations of (1.1) in the interior of $D$.

Definition II. We will say that the function $F_{s}\left(x, t, y_{i}, z_{j}, z_{j k}\right)$ satisfies the (I) -condition if there exist positive constants $L_{0}, \ldots, L_{\mathbf{4}}$ such that, for arbitrary $y_{i}, z_{j}, z_{j k}, \bar{y}_{i}, \bar{z}_{j}, \bar{z}_{j k}(i=1, \ldots, n ; j, k=1, \ldots, m)$, $y_{s} \geqslant \bar{y}_{s}$, we have the inequality

$$
\begin{aligned}
& \boldsymbol{F}_{8}\left(x, t, y_{i}, z_{j}, z_{j k}\right)-\boldsymbol{F}_{s}\left(x, t, \bar{y}_{i}, \bar{z}_{j}, \bar{z}_{j k}\right) \\
& \leqslant L_{0} \sum_{j, k=1}^{m}\left|z_{j k}-\bar{z}_{j k}\right|+\left(L_{1}|x|+L_{2}\right) \sum_{j=1}^{m}\left|z_{j}-\bar{z}_{j}\right|+\left(L_{\mathbf{3}}|x|^{2}+L_{4}\right) \sum_{i=1}^{n}\left|y_{i}-\bar{y}_{i}\right|,
\end{aligned}
$$


where $|x|=\left(\sum_{i=1}^{m} x_{1}^{2}\right)^{1 / 2}$. It means that the function $F_{8}$ satisfies the Lipschitz condition with respect to the variables $y_{1}, \ldots, y_{s-1}, y_{s+1}, \ldots, y_{n}, z_{j}, z_{j k}$ $(j, k=1, \ldots, m)$ (at the same time some of the Lipschitz constants depend on $x$ ) and that the difference quotient of $F_{s}$ for the variable $y_{8}$ is bounded only from above by $L_{3}|x|^{2}+L_{4}$.

By $E_{a}(M, K)$ or shortly $E_{\delta}$ we will denote the class of functions $\psi(x, t)$ for which positive constants $M, K$ exist such that the inequality

$$
|\psi(x, t)| \leqslant M \exp \left(K|x|^{\delta}\right)
$$

is satisfied in $D$, and by $Z_{\delta}(A, B)$ or $Z_{\delta}, \delta>0$, we will denote the class of functions $\chi(x, t)$ for which positive constants $A, B$ exist such that

$$
|\chi(x, t)| \leqslant A|x|^{\delta}+B \quad \text { for } \quad(x, t) \in D .
$$

§ 2. Formulation of the problem. Let $\varphi_{i}(x, t)(i=1, \ldots, n)$ be arbitrary given functions, defined and continuous in the set $\Sigma=\boldsymbol{S}^{0}+\sigma$.

In this paper we discuss the first boundary-value problem of Fourier, which we formulate as follows: to find a parabolic solution $u_{i}(x, t)$ $(i=1, \ldots, n)$ of system (1.1), regular in $D$ and such that

$$
u_{i}(x, t)=\varphi_{i}(x, t) \quad(i=1, \ldots, n) \quad \text { for } \quad(x, t) \in \Sigma .
$$

In what follows we will call this problem the (FI)-problem.

\$3. The uniqueness of solution of the (FI)-problem.

THEOREM I. If each function $\boldsymbol{F}_{s}\left(x, t, y_{i}, z_{j}, z_{j k}\right)(s=1, \ldots, n)$ fulfils the (I)-condition in $I I$, then the (FI)-problem possesses no more than one solution $u_{1}(x, t), \ldots, u_{n}(x, t)$ of class $E_{2}{ }^{\left({ }^{1}\right)}$ in the domain $D$.

Proof. Suppose there exist two such solutions, $u_{i}^{(1)}$ and $u_{i}^{(2)}(i=1, \ldots, n)$. Then the differences $u_{i}=u_{i}^{(1)}-u_{i}^{(2)}$ satisfy the system of equations

$$
\begin{aligned}
& \frac{\partial u_{s}}{\partial t}=F_{s}\left(x, t, u_{i}^{(1)}, \frac{\partial u_{s}^{(1)}}{\partial x_{j}}, \frac{\partial^{2} u_{s}^{(1)}}{\partial x_{j} \partial x_{k}}\right)-F_{s}\left(x, t, u_{i}^{(2)}, \frac{\partial u_{s}^{(2)}}{\partial x_{j}}, \frac{\partial^{2} u_{s}^{(2)}}{\partial x_{j} \partial x_{k}}\right) \\
& (s=1, \ldots, n) \text {. }
\end{aligned}
$$

We may assume that all the functions $u_{i}(i=1, \ldots, n)$ belong to $E_{2}\left(M, K_{0}\right)$ with the same constants $M$ and $K_{0}$.

Let $D_{R}$ be an open bounded set separated from $D$ by the cylindrical surface $\Gamma_{R}$ with the equation $\sum_{i=1}^{m} x_{i}^{2}=R^{2}$.

We will denote by $S_{R}^{0}, S_{R}^{T}$ and $\Sigma_{R}$ the parts of the surfaces $S^{0}, S^{T}$ and $\Sigma$, respectively, lying inside and on $\Gamma_{R}$, whereas by $C_{R}$ we will de-

(1) I.e. each function $u_{i}(x, t)(i=1, \ldots, n)$ belongs to $E_{2}^{1}$. 
note the part of $\Gamma_{R}$ contained in $D$. Further, let $D^{h}, D_{R}^{h}, \Sigma^{h}, \Sigma_{R}^{h}, C_{R}^{h}$ denote the parts of the sets $D, D_{R}, \Sigma, \Sigma_{R}, C_{R}$, respectively, contained in the strip $0 \leqslant t<h, h \leqslant T$.

We first prove our theorem for a domain $D^{h}$, where $h$ will be a conveniently chosen (sufficiently small) constant.

Now we introduce the functions $v_{i}^{(1)}, v_{i}^{(2)}$ according to the relations

$$
u_{i}^{(1)}=v_{i}^{(1)} H(x, t ; K), \quad u_{i}^{(1)}=v_{i}^{(2)} H(x, t ; K) \quad(i=1, \ldots, n),
$$

where

$$
H(x, t ; K)=\exp \left\{\frac{K|x|^{2}}{1-\mu t}+\nu t\right\}
$$

is the auxiliary function constructed by M. Krzyżański in order to prove similar theorems for the linear parabolic equation (see, for instance, [6]). Here $\mu(K)$ and $\nu(K)$ are positive constants which will be conveniently chosen for $K>K_{0}$.

Writing $v_{i}=v_{i}^{(1)}-v_{i}^{(2)}$ we have $u_{i}=v_{i} H(x, t ; K)$ in $D^{h}$ where $h<1 / \mu(K)$. Introducing the functions $v_{i}, v_{i}^{(1)}, v_{i}^{(2)}$ into (3.1), subtracting and adding convenient expressions we get the system

$$
\begin{aligned}
& F_{s}\left(x, t, v_{i}^{(1)} H, \frac{\partial v_{s}^{(1)}}{\partial x_{j}} H+v_{s}^{(1)} \frac{\partial H}{\partial x_{j}}, \frac{\partial^{2} v_{s}^{(1)}}{\partial x_{j} \partial x_{k}} H+\frac{\partial v_{s}^{(1)}}{\partial x_{k}} \frac{\partial H}{\partial x_{j}}+\frac{\partial v_{s}^{(1)}}{\partial x_{j}} \frac{\partial H}{\partial x_{k}}+v_{s}^{(1)} \frac{\partial^{2} H}{\partial x_{j} \partial x_{k}}\right)- \\
& -F_{s}\left(x, t, v_{i}^{(1)} H, \frac{\partial v_{s}^{(1)}}{\partial x_{j}} H+v_{s}^{(1)} \frac{\partial H}{\partial x_{j}}, \frac{\partial^{2} v_{s}^{(2)}}{\partial x_{j} \partial x_{k}} H+\frac{\partial v_{s}^{(1)}}{\partial x_{k}} \frac{\partial H}{\partial x_{j}}+\frac{\partial v_{s}^{(1)}}{\partial x_{j}} \frac{\partial H}{\partial x_{k}}+v_{s}^{(1)} \frac{\partial^{2} H}{\partial x_{j} \partial x_{k}}\right)+ \\
& +F_{s}\left(x, t, v_{i}^{(1)} H, \frac{\partial v_{s}^{(1)}}{\partial x_{j}} H+v_{s}^{(1)} \frac{\partial H}{\partial x_{j}}, \frac{\partial^{2} v_{s}^{(2)}}{\partial x_{j} \partial x_{k}} H+\frac{\partial v_{s}^{(1)}}{\partial x_{k}} \frac{\partial H}{\partial x_{j}}+\frac{\partial v_{s}^{(1)}}{\partial x_{j}} \frac{\partial H}{\partial x_{k}}+v_{s}^{(1)} \frac{\partial^{2} H}{\partial x_{j} \partial x_{k}}\right)- \\
& -F_{s}\left(x, t, v_{i}^{(2)} H, \frac{\partial v_{s}^{(2)}}{\partial x_{j}} H+v_{s}^{(2)} \frac{\partial H}{\partial x_{j}}, \frac{\partial^{2} v_{s}^{(2)}}{\partial x_{j} \partial x_{k}} H+\frac{\partial v_{s}^{(2)}}{\partial x_{k}} \frac{\partial H}{\partial x_{j}}+\frac{\partial v_{s}^{(2)}}{\partial x_{j}} \frac{\partial H}{\partial x_{k}}+v_{s}^{(2)} \frac{\partial^{2} H}{\partial x_{j} \partial x_{k}}\right) \\
& (s=1, \ldots, n) .
\end{aligned}
$$

Let $\left\{R_{a}\right\}$ be an increasing sequence, $R_{a} \rightarrow \infty$ as $\alpha \rightarrow \infty$. Let us consider (for a fixed $a$ ) the domain $D_{R_{a}}^{h}$. We write

$$
A_{a}=\max _{(i)} \sup _{(x, l) \in \bar{D}_{R_{a}}^{h}}\left|v_{i}(x, t)\right|
$$

There exist an index $i_{a}$ and a point $\left(x_{a}, t_{a}\right) \in \overline{D_{R_{a}}^{h}}=\left(D_{R_{a}}^{h}+S_{R_{a}}^{h}\right)+\Sigma_{R_{a}}^{h}+C_{R_{a}}^{h}$ such that $A_{a}=\left|v_{i_{a}}\left(x_{a}, t_{a}\right)\right|$. We will show that, the constants $\mu(K), v(K)$ and $h(K)$ being conveniently chosen, the relation $\left(x_{a}, t_{a}\right) \in D_{R_{a}}^{h}+S_{R_{a}}^{h}$ 
implies $v_{i_{a}}\left(x_{a}, t_{a}\right)=0$. Indeed, suppose the contrary; there are two cases to be considered:

a) $v_{i_{a}}\left(x_{a}, t_{a}\right)>0$. Then at $\left(x_{a}, t_{a}\right)$ we have

$$
\frac{\partial v_{i_{a}}}{\partial t} \geqslant 0, \quad \frac{\partial v_{i_{a}}}{\partial x_{f}}=0 \quad(j=1, \ldots, m) \quad \text { and } \quad \sum_{j, k=1}^{m} \frac{\partial^{2} v_{i_{a}}}{\partial x_{j} \partial x_{k}} \lambda_{j} \lambda_{k} \leqslant 0 .
$$

Substituting $x=x_{a}, t=t_{a}$ in the equation with the index $i_{a}$ of (3.3) and taking into account the definition of parabolic equation (see $\S 1$ ), we find that the difference of the first two terms on the right side of this equation is non-positive. Now we estimate the difference of the remaining two terms. According to the (R)-condition assumed we have

$$
\begin{aligned}
\frac{\partial v_{i_{a}}}{\partial t} H+v_{i_{a}} \frac{\partial H}{\partial t} \leqslant & v_{i_{a}} L_{0} \sum_{j, k=1}^{m}\left|\frac{\partial^{2} H}{\partial x_{j} \partial x_{k}}\right|+ \\
& +v_{i_{a}}\left(L_{1}|x|+L_{2}\right) \sum_{j=1}^{m}\left|\frac{\partial H}{\partial x_{j}}\right|+\left(L_{3}|x|^{2}+L_{4}\right) \sum_{j=1}^{n}\left|v_{i}\right| H .
\end{aligned}
$$

Since $\sum_{j=1}^{n}\left|v_{i}\left(x_{\alpha}, t_{\alpha}\right)\right| \leqslant n \cdot v_{i_{a}}\left(x_{\alpha}, t_{\sigma}\right)$, we have

$$
\begin{aligned}
\frac{\partial v_{i_{a}}}{\partial t} H \leqslant & v_{i_{a}}\left\lceil L_{0} \sum_{j, k=1}^{m}\left|\frac{\partial^{2} H}{\partial x_{j} \partial x_{k}}\right|+\right. \\
& \left.+\left(L_{1}|x|+L_{2}\right) \sum_{j=1}^{m}\left|\frac{\partial H}{\partial x_{j}}\right|+\left(L_{3}|x|^{2}+L_{4}\right) n H-\frac{\partial H}{\partial t}\right] \stackrel{d f}{=} v_{i_{a}} F H .
\end{aligned}
$$

We will show, as in paper [6], that the constants $\mu(K), \nu(K)$ and $h(K)$ can be chosen (independently of $\alpha$ ) in such a way that $F H \leqslant-N H$, $N$ being an arbitrary positive number. Namely, we have

$$
\begin{aligned}
F H \leqslant H\left[\frac{4 K^{2} L_{0}}{(1-\mu t)^{2}} \sum_{j, k=1}^{m}\left|x_{j} x_{k}\right|+\frac{2 K L_{0} m}{1-\mu t}\right. & +\frac{2 K}{1-\mu t}\left(L_{1}|x|+L_{2}\right) \sum_{j=1}^{m}\left|x_{j}\right|+ \\
& \left.+\left(L_{3}|x|^{2}+L_{4}\right) n-\frac{\mu K|x|^{2}}{(1-\mu t)^{2}}-\nu\right] .
\end{aligned}
$$

Writing $L=\max \left(L_{1}, L_{2}\right)$ and taking into consideration the inequalities $\left|x_{j}\right| \leqslant|x|,|x| \leqslant|x|^{2}+1,1-\mu t \leqslant 1$, we have

$$
\begin{aligned}
F H \leqslant \frac{H}{(1-\mu t)^{2}}\left[4 K^{2} L_{0} m^{2}|x|^{2}+2 K L_{0} m+4 K L m|x|^{2}+\right. \\
\left.+2 K L m+n L_{3}|x|^{2}+n L_{4}-\mu K|x|^{2}-\nu(1-\mu t)^{2}\right] .
\end{aligned}
$$




\section{Choosing}

$$
\begin{aligned}
& \mu(K)=4 K L_{0} m^{2}+4 L m+\frac{n L_{3}+\lambda}{K} \quad(\lambda \text {-arbitrary positive constant }) \\
& h(K)=\frac{1-\gamma}{\mu(K)}, \quad 0<\gamma<1
\end{aligned}
$$

we obtain $\gamma \leqslant 1-\mu t \leqslant 1$ in $D^{h}$, and hence

$$
F H \leqslant \frac{H}{(1-\mu t)^{2}}\left[-\lambda|x|^{2}+2 K L_{0} m+2 K L m+n L_{4}-v \gamma^{2}\right] .
$$

\section{Putting}

$$
v(K)=\frac{2 K L_{0} m+2 K L m+n L_{4}+N}{\gamma^{2}} \quad(N-\text { arbitrary positive constant })
$$

we have

$$
F H \leqslant-\lambda H|x|^{2}-N H \leqslant-N H<0 .
$$

This is in contradiction with $\frac{\partial v_{i_{a}}\left(x_{a}, t_{a}\right)}{\partial t} \geqslant 0$ and (3.4).

b) $v_{i_{a}}\left(x_{a}, t_{a}\right)<0$. Repeating the above reasoning for the function $\bar{v}_{i_{a}}(x, t)=-v_{\iota_{a}}(x, t)$, we also obtain a contradiction. Now, if $\left(x_{a}, t_{a}\right) \in \Sigma_{R_{a}}^{h}$, we have (by our assumption) $v_{t_{a}}\left(x_{a}, t_{a}\right)=0$, and finally if $\left(x_{a}, t_{a}\right) \in C_{R_{a}}^{h}$, then

$$
\left|v_{i_{a}}\left(x_{a}, t_{a}\right)\right| \leqslant \frac{M \exp \left(K_{0}\left|x_{a}\right|^{2}\right)}{\exp \left\{\frac{K\left|x_{a}\right|^{2}}{1-\mu t_{a}}+\nu t_{a}\right\}}=\frac{M \exp \left(K_{0} R_{a}^{2}\right)}{\exp \left\{\frac{K R_{a}^{2}}{1-\mu t_{a}}+v t_{a}\right\}}
$$

Thus, since $\left|v_{i}(x, t)\right| \leqslant\left|v_{i_{a}}\left(x_{a}, t_{a}\right)\right|$ in $\overline{D_{R_{a}}^{h}}$, we have proved that, the constants $\mu(K), \nu(K)$ and $h(K)$ being chosen according to (3.5) and (3.6), we have in $\overline{D_{R_{\alpha}}^{h}}$ the inequalities

$$
\left|v_{i}(x, t)\right| \leqslant \frac{M \exp \left(K_{0} R_{a}^{2}\right)}{\exp \left\{\frac{K R_{a}^{2}}{1-\mu t_{a}}+\nu t_{a}\right\}} \quad(i=1, \ldots, n) .
$$

Now let us take an arbitrary point $(\bar{x}, \bar{t}) \in \overline{D^{h}}$. For an arbitrary number $\varepsilon>0$ there exists an index $\alpha_{0}(\varepsilon)$ such that for $\alpha>\alpha_{0}$ the point $(\bar{x}, \bar{t}) \epsilon \bar{D}_{\boldsymbol{R}_{a}}^{h}$ and

$$
\frac{M \exp \left(K_{0} R_{a}^{2}\right)}{\exp \left\{\frac{K R_{a}^{2}}{1-\mu t_{a}}+\nu t_{\alpha}\right\}}<\varepsilon .
$$


Consequently, $v_{i}(\bar{x}, \bar{t})=0$, whence $u_{i}(\bar{x}, \bar{t})=0$ and thus $u_{i}(x, t)=0$ $(i=1, \ldots, n)$ in the whole domain $\overline{D^{h}}$.

Similarly, the theorem can be proved for the parts of the domain $D$ contained in the strips: $j h \leqslant t \leqslant(j+1) h(j=1,2, \ldots)$. For this purpose it is sufficient to put $t=\tilde{t}+j \cdot h(j=1,2, \ldots)$ (see, for instance, [9]).

§ 4. Centrain inequalities between solutions of the (FI) problem for two systems of equations. Under a supplementary assumption we will prove a stronger theorem than theorem $\mathbf{I}$.

Let us take a system of functions $\psi_{1}\left(y_{1}, \ldots, y_{n}, \tau\right), \ldots, \psi_{n}\left(y_{1}, \ldots, y_{n}, \tau\right)$, where $\tau$ denotes a sequence of variables different from $y_{1}, \ldots, y_{n}$. The following definition is introduced: the function $\psi_{s}\left(y_{1}, \ldots, y_{n}, \tau\right)$ satisfies the (W)-condition with regard to variables $y_{1}, \ldots, y_{n}$ if for $y_{i} \leqslant \bar{y}_{1}, i \neq s$, $y_{s}=\bar{y}_{s}$ we have the inequality

$$
\psi_{s}\left(y_{1}, \ldots, y_{n}, \tau\right) \leqslant \psi_{s}\left(\bar{y}_{1}, \ldots, \bar{y}_{n}, \tau\right) \text {. }
$$

THEOREM II. $1^{0}$ Let $u_{i}^{(1)}(x, t), u_{i}^{(2)}(x, t)(i=1, \ldots, n)$ be regular solutions, belonging to class $E_{2}$, of the systems of equations

$$
\begin{array}{ll}
\frac{\partial u_{s}^{(1)}}{\partial t}=F_{s}^{(1)}\left(x, t, u_{i}^{(1)}, \frac{\partial u_{s}^{(1)}}{\partial x_{j}}, \frac{\partial^{2} u_{s}^{(1)}}{\partial x_{j} \partial x_{k}}\right) & (s=1, \ldots, n), \\
\frac{\partial u_{s}^{(2)}}{\partial t}=F_{s}^{(2)}\left(x, t, u_{i}^{(2)}, \frac{\partial u_{s}^{(2)}}{\partial x_{j}}, \frac{\partial^{2} u_{s}^{(2)}}{\partial x_{j} \partial x_{k}}\right) \quad(s=1, \ldots, n) .
\end{array}
$$

$2^{\circ}$ For each $s(s=1, \ldots, n)$ the equation with the index $s$ of system (4.1) or (4.2) is parabolic (see §1) with regard to the solution $u_{s}^{(1)}(x, t)$ and $u_{s}^{(2)}(x, t)$, respectively.

$3^{\circ}$ For each $s \quad(s=1, \ldots, n)$ the function $F_{s}^{(1)}\left(x, t, y_{i}, z_{j}, z_{j k}\right)$ or $F_{s}^{(2)}\left(x, t, y_{i}, z_{j}, z_{j k}\right)$ satisfies the (W)-condition with respect to the variables $y_{1}, \ldots, y_{n}$ and the (I)-condition (independently of the respective equation of the system (4.1) or (4.2) being parabolic).

$\mathbf{4}^{\mathbf{0}} F_{s}^{(1)}\left(x, t, y_{i}, z_{j}, z_{j k}\right) \leqslant F_{s}^{(2)}\left(x, t, y_{i}, z_{j}, z_{j k}\right)(s=1, \ldots, n)$ for $\left(x, t, y_{i}\right.$, $\left.z_{j}, z_{j k}\right) \in \Pi$.

$5^{\mathrm{o}} u_{i}^{(1)}(x, t) \leqslant u_{i}^{(2)}(x, t)(i=1, \ldots, n)$ for $(x, t) \in \Sigma$.

Under the assumptions $1^{\circ}-5^{\circ}$ the inequalities

$$
u_{i}^{(1)}(x, t) \leqslant u_{i}^{(())}(x, t) \quad(i=1, \ldots, n)
$$

hold for $(x, t) \in \bar{D}$.

Proof. For simplification we may assume that all the equations of system (4.2) are parabolic with respect to the solution $u_{i}^{(2)}(x, t)$ and that all the functions $F_{s}^{(2)}(s=1, \ldots, n)$ satisfy the $(\mathfrak{L})$ - and (W)-conditions. In the remaining cases the proof is similar. 
The differences $u_{i}=u_{i}^{(1)}-u_{i}^{(2)}$ belong to the class $E_{2}\left(M, K_{0}\right)$. Let us introduce the functions $v_{i}^{(1)}, v_{i}^{(2)}$ according to the relations $u_{i}^{(1)}=v_{i}^{(1)} H$, $u_{i}^{(2)}=v_{i}^{(2)} H \quad(i=1, \ldots, n)$, where $H=H(x, t ; K), K>K_{0}$, is defined by (3.2). Writing $v_{i}=v_{i}^{(1)}-v_{i}^{(2)}$, we obtain $u_{i}=v_{i} H$.

Let us put

$$
\begin{aligned}
\tau_{j}^{(1)} & =\frac{\partial v_{s}^{(1)}}{\partial x_{j}} H+v_{s}^{(1)} \frac{\partial H}{\partial x_{j}}, \quad \tau_{j}^{(2)}=\frac{\partial v_{s}^{(2)}}{\partial x_{j}} H+v_{s}^{(2)} \frac{\partial H}{\partial x_{j}}, \\
\tau_{j k}^{(1,1)} & =\frac{\partial^{2} v_{s}^{(1)}}{\partial x_{j} \partial x_{k}} H+\frac{\partial v_{s}^{(1)}}{\partial x_{j}} \frac{\partial H}{\partial x_{k}}+\frac{\partial v_{s}^{(1)}}{\partial x_{k}} \frac{\partial H}{\partial x_{j}}+v_{s}^{(1)} \frac{\partial^{2} H}{\partial x_{j} \partial x_{k}}, \\
\tau_{j k}^{(1,2)} & =\frac{\partial^{2} v_{s}^{(1)}}{\partial x_{j} \partial x_{k}} H+\frac{\partial v_{s}^{(2)}}{\partial x_{j}} \frac{\partial H}{\partial x_{k}}+\frac{\partial v_{s}^{(2)}}{\partial x_{k}} \frac{\partial H}{\partial x_{j}}+v_{s}^{(2)} \frac{\partial^{2} H}{\partial x_{j} \partial x_{k}}, \\
\tau_{j k}^{(2,2)} & =\frac{\partial^{2} v_{s}^{(2)}}{\partial x_{j} \partial x_{k}} H+\frac{\partial v_{s}^{(2)}}{\partial x_{j}} \frac{\partial H}{\partial x_{k}}+\frac{\partial v_{s}^{(2)}}{\partial x_{k}} \frac{\partial H}{\partial x_{j}}+v_{s}^{(2)} \frac{\partial^{2} H}{\partial x_{j} \partial x_{k}} .
\end{aligned}
$$

Subtracting the adequate equations of (4.2) from (4.1), introducing the functions $v_{i}^{(1)}, v_{i}^{(2)}, v_{i}$, and subtracting and adding convenient expressions, we receive:

$$
\begin{aligned}
& \frac{\partial v_{s}}{\partial t} H+v_{s} \frac{\partial H}{\partial t}= F_{s}^{(1)}\left(x, t, v_{i}^{(1)} H, \tau_{j}^{(1)}, \tau_{j k}^{(1,1)}\right)-F_{s}^{(2)}\left(x, t, v_{i}^{(1)} H, \tau_{j}^{(1)}, \tau_{j k}^{(1,1)}\right)+ \\
&+F_{s}^{(2)}\left(x, t, v_{i}^{(2)} H, \tau_{j}^{(2)}, \tau_{j k}^{(1,2)}\right)-F_{s}^{(2)}\left(x, t, v_{i}^{(2)} H, \tau_{j}^{(2)}, \tau_{j k}^{(2,2)}\right)+ \\
&+F_{s}^{(2)}\left(x, t, v_{i}^{(1)} H, \tau_{j}^{(1)}, \tau_{j k}^{(1,1)}\right)-F_{s}^{(2)}\left(x, t, v_{i}^{(2)} H, \tau_{j}^{(2)}, \tau_{j k}^{(1,2)}\right) \\
&(s=1, \ldots, n)
\end{aligned}
$$

Let us take an increasing sequence $\left\{R_{\alpha}\right\}, R_{\alpha} \rightarrow \infty$ as $\alpha \rightarrow \infty$. We retain the meaning of the symbols $D^{h}, D_{R}^{h}, \ldots, C_{R}^{h}$ introduced in the proof of theorem I. Let us denote

$$
\bar{A}_{a}=\max _{(i)} \sup _{(x, l) \in \overline{D_{R_{a}}^{h}}} v_{i}(x, t) .
$$

There exist an index $i_{a}$ and a point $\left(x_{\alpha}, t_{\alpha}\right) \in \overline{D_{H_{a}}^{h}}=\left(D_{R_{a}}^{h}+S_{R_{a}}^{h}\right)+\sum_{R_{a}}^{h}+C_{R_{a}}^{h}$ such that $\bar{A}_{\alpha}=v_{i_{a}}\left(x_{a}, t_{a}\right)$. We will show that, the constants $\mu(K), v(K)$ and $h(K)$ being conveniently chosen, the relation $\left(x_{a}, t_{a}\right) \in D_{R_{a}}^{h}+S_{R_{a}}^{h}$ implies the inequality $v_{i_{a}}\left(x_{a}, t_{a}\right) \leqslant 0$. Indeed, suppose that $\left(x_{\alpha}, t_{a}\right) \in D_{R_{a}}^{h}+$ $+S_{R_{a}}^{h}$ and $v_{i_{a}}\left(x_{a}, t_{a}\right)>0$. Then at $\left(x_{a}, t_{a}\right)$ we have

$$
\frac{\partial v_{i_{a}}}{\partial t} \geqslant 0, \quad \frac{\partial v_{i_{a}}}{\partial x_{j}}=0 \quad \text { as well as } \quad \sum_{j, k=1}^{m} \frac{\partial^{2} v_{i_{a}}}{\partial x_{j}} \frac{\partial}{\partial x_{k}} \lambda_{j} \lambda_{k} \leqslant 0 .
$$


Substituting $x=x_{a}, t=t_{a}$ in the equation with the index $i_{a}$ of (4.4) we find, by virtue of assumption $4^{\circ}$, that the difference of the first two terms on the right side of this equation is non-positive, and from the definition of the parabolic equations it follows that the difference of the next two terms is also non-positive.

Suppose that a certain number (e.g. $q, 1 \leqslant q \leqslant n$ ) of the functions among $v_{1}, \ldots, v_{n}$ at point $\left(x_{a}, t_{a}\right)$ admit a positive value the others having non-positive values. Without loss of generality we may assume that they are the functions $v_{1}, \ldots, v_{q}$ (and that $i_{a} \leqslant q$ ).

The difference of the last two terms in the equation with the index $i_{a}$ of (4.4) at $\left(x_{a}, t_{a}\right)$ can be written as

$$
\begin{aligned}
& F_{i_{a}}^{(2)}\left(x_{a}, t_{a}, v_{1}^{(1)} H, \ldots, v_{q}^{(1)} H, v_{q+1}^{(1)} H, \ldots, v_{n}^{(1)} H, \tau_{j}^{(1)}, \tau_{j k}^{(1,1)}\right)- \\
- & F_{i_{a}}^{(2)}\left(x_{a}, t_{a}, v_{1}^{(2)} H, \ldots, v_{q}^{(2)} H, v_{q+1}^{(1)} H, \ldots, v_{n}^{(1)} H, \tau_{j}^{(2)}, \tau_{j k}^{(1,2)}\right)+ \\
+ & F_{i_{a}}^{(2)}\left(x_{a}, t_{a}, v_{1}^{(2)} H, \ldots, v_{q}^{(2)} H, v_{q+1}^{(1)} H, \ldots, v_{n}^{(1)} H, \tau_{j}^{(2)}, \tau_{j k}^{(1,2)}\right)- \\
- & F_{i_{a}}^{(2)}\left(x_{a}, t_{a}, v_{1}^{(2)} H, \ldots, v_{q}^{(2)} H, v_{q+1}^{(2)} H, \ldots, v_{n}^{(2)} H, \tau_{j}^{(2)}, \tau_{j k}^{(1,2)}\right) .
\end{aligned}
$$

By the (W)-condition the difference of the last two terms in (4.5) is nonpositive; however, the difference of the first two terms is estimated by virtue of the (2)-condition. Taking at the same time into consideration the equalities

we have

$$
\frac{\partial v_{i_{a}}^{(1)}\left(x_{a}, t_{a}\right)}{\partial x_{j}}=\frac{\partial v_{i_{a}}^{(2)}\left(x_{a}, t_{\alpha}\right)}{\partial x_{j}} \quad(j=1, \ldots, m),
$$

$$
\begin{aligned}
0 \leqslant \frac{\partial v_{i_{a}}}{\partial t} H \leqslant v_{i_{a}}\left[L_{0} \sum_{j, k=1}^{m}\left|\frac{\partial^{2} H}{\partial x_{j} \partial x_{k}}\right|+\right. & \left(L_{1}|x|+L_{2}\right) \sum_{j=1}^{m}\left|\frac{\partial H}{\partial x_{j}}\right|+ \\
& \left.+\left(L_{3}|x|^{2}+L_{4}\right) q H-\frac{\partial H}{\partial t}\right] \stackrel{\text { df }}{=} v_{i_{a}} F_{1} H .
\end{aligned}
$$

Choosing conveniently $\mu(K), \nu(K)$ and $h(K)$, as in the proof of theorem I, we find $F_{1} H \leqslant-N H<0$. Thus we obtain a contradiction. Now, if $\left(x_{a}, t_{a}\right) \in \Sigma_{R_{a}}^{h}$, then (by our assumption) $v_{i_{a}}\left(x_{a}, t_{a}\right) \leqslant 0$ and finally, if $\left(x_{a}, t_{a}\right) \in C_{R_{a}}^{h}$, then we have

$$
v_{i_{\alpha}}\left(x_{\alpha}, t_{\alpha}\right) \leqslant \frac{M \exp \left(K_{0} R_{a}^{2}\right)}{\exp \left\{\frac{K R_{a}^{2}}{1-\mu t_{\alpha}}+v t_{\alpha}\right\}}
$$

Thus we have proved that, the constants $\mu(K), \nu(K)$ and $h(K)$ being conveniently chosen, the last inequality always holds. 
Let $(\bar{x}, \bar{t})$ be an arbitrary fixed point of $\overline{D^{h}}$. For every $\varepsilon>0$ there exists such an $\alpha_{0}(\varepsilon)$ that for $a>\alpha_{0}$ the point $(\bar{x}, \bar{t}) \epsilon \overline{D_{R_{a}}^{h}}$ and

Since

$$
\frac{M \exp \left(K_{0} R_{a}^{2}\right)}{\exp \left\{\frac{K R_{a}^{2}}{1-\mu t_{a}}+\nu t_{a}\right\}}<\varepsilon .
$$

$$
v_{i}(\bar{x}, \bar{t}) \leqslant v_{i_{a}}\left(x_{a}, t_{a}\right) \leqslant \frac{M \exp \left(K_{0} R_{a}^{2}\right)}{\exp \left\{\frac{K R_{a}^{2}}{1-\mu t_{\alpha}}+\nu t_{a}\right\}} \quad(i=1, \ldots, n),
$$

we have $v_{i}(\bar{x}, \bar{t}) \leqslant 0(i=1, \ldots, n)$, whence $v_{i}(x, t) \leqslant 0$ and thus $u_{i}(x, t) \leqslant 0$ $(i=1, \ldots, n)$ everywhere in $\overline{D^{h}}$.

As in the proof of theorem $\mathrm{I}$, the change of variable $t$ permits to prove this theorem in the whole domain $D$.

Remark. It is easy to observe that the change of direction of the inequalities in assumptions $4^{\circ}$ and $5^{\circ}$ changes the direction of the inequalities in our theorem. If, therefore, $F_{s}^{(1)} \equiv F_{s}^{(2)}$ in $\Pi$ and $u_{i}^{(1)}(x, t)$ $=u_{i}^{(2)}(x, t)$ in $\Sigma$, then $u_{i}^{(1)}(x, t) \equiv u_{i}^{(2)}(x, t)$ in $\bar{D}$. Hence we obtain the uniqueness of the solution of the (FI)-problem, under stronger assumption, however, than in theorem I. In the case of one equation, theorem II is more general than theorem I because then the (W)-condition does not interfere.

We have proved theorem II in the case where all the equations of (4.2) are parabolic and all the functions $F_{s}^{(2)}(s=1, \ldots, n)$ satisfy the $(\mathfrak{L})$-condition and the (W)-condition. In this case the equations of (4.1) are not necessarily parabolic, just as the adequate functions do not necessarily satisfy either the $(\mathfrak{L})$ - or the $(\mathrm{W})$-conditions. For example, the functions $u_{1}^{(1)}=\pi / 2+e^{-\left(x_{1}+x_{2}\right)}, u_{2}^{(1)}=\pi / 2$ are the solution of the system

$$
\begin{aligned}
& \frac{\partial u_{1}^{(1)}}{\partial t}=\frac{\partial^{2} u_{2}^{(1)}}{\partial x_{1}^{2}}-\left|\frac{\partial^{2} u_{1}^{(1)}}{\partial x_{2}^{2}}\right|+\sqrt{\mid \sin u_{2}^{(1)}}-1, \\
& \frac{\partial u_{2}^{(1)}}{\partial t}=-\left|\frac{\partial^{2} u_{2}^{(1)}}{\partial x_{1}^{2}}\right|+\frac{\partial^{2} u_{2}^{(1)}}{\partial x_{2}^{2}}-\sqrt{\left|\overline{u_{1}^{(1)}-u_{2}^{(1)}}\right|}+u_{1}^{(1)}-u_{2}^{(1)}+e^{-\frac{x_{1}+x_{2}}{2}}-e^{-\left(x_{1}+x_{2}\right)},
\end{aligned}
$$

whereas the functions $u_{1}^{(2)}=3, u_{2}^{(2)}=5$ constitute the solution of the system

$$
\begin{aligned}
& \frac{\partial u_{1}^{(2)}}{\partial t}=\frac{\partial^{2} u_{1}^{(2)}}{\partial x_{1}^{2}}+\frac{\partial^{2} u_{1}^{(2)}}{\partial x_{2}^{2}}, \\
& \frac{\partial u_{2}^{(2)}}{\partial t}=\frac{\partial^{2} u_{2}^{(2)}}{\partial x_{1}^{2}}+\frac{\partial^{2} u_{2}^{(2)}}{\partial x_{2}^{2}}+u_{1}^{(2)}-u_{2}^{(2)}+2 .
\end{aligned}
$$


The inequalities $u_{1}^{(1)}<u_{2}^{(2)}, u_{2}^{(1)}<u_{2}^{(2)}$ are fulfilled in the part of space $x_{1} \geqslant 0, x_{2} \geqslant 0, t \geqslant 0$. All the assumptions of theorem II are satisfied. The equations of (4.6) are not parabolic and, moreover, the suitable functions do not satisfy either the $(\mathcal{L})$ - or the (W)-condition.

Corollary. In the class $Z_{\boldsymbol{\delta}}$ (see $\left.\S 1\right)$ of functions $u_{\imath}(x, t)(i=1, \ldots, n)$, $\delta$ being an arbitrary positive constant, we can prove theorems I and II if the (R)-condition is replaced by a weaker one. Namely, it is sufficient to assume that there exist positive constants $L_{0}, L_{1}, \ldots, L_{8}$ such that for $y_{s} \geqslant \bar{y}_{s}$ $(s=1, \ldots, n)$ the following inequalities hold:

$$
\begin{aligned}
& \boldsymbol{F}_{s}\left(x, t, y_{i}, z_{j}, z_{j k}\right)-F_{s}\left(x, t, \bar{y}_{i}, \bar{z}_{j}, \bar{z}_{j k}\right) \\
& \leqslant L_{0} \sum_{j, k=1}^{m}\left|z_{j k}-\bar{z}_{j k}\right|+\left(L_{1}|x|+L_{2}|y|^{1 / \delta}+L_{3} \mid \bar{y}^{\mid 1 / \delta}+L_{4}\right) \sum_{j=1}^{m}\left|z_{j}-\bar{z}_{j}\right|+ \\
& +\left(L_{5}|x|^{2}+L_{6}|y|^{2 / \delta}+L_{7}|\bar{y}|^{2 / \delta}+L_{8}\right) \sum_{i=1}^{n}\left|y_{i}-\bar{y}_{i}\right|,
\end{aligned}
$$

where

$$
|y|=\left(\sum_{i=1}^{n} y_{i}^{2}\right)^{1 / 2}, \quad|\bar{y}|=\left(\sum_{i=1}^{n} \bar{y}_{i}^{2}\right)^{1 / 2} .
$$

Proof. If there exist positive constants $A, B$ such that $\left|y_{i}\right| \leqslant A|x|^{\delta}+$ $+B$, then

$$
|y|^{1 / \delta}=\left(\sum_{i=1}^{n}\left|y_{i}\right|^{2}\right)^{1 / 2 \delta} \leqslant n^{1 / 2 \delta}\left(A|x|^{\delta}+B\right)^{1 / \delta} .
$$

Put $A_{1} \stackrel{\text { df }}{=} n^{1 / 28}(A+B)^{1 / \delta}$. In the case $|x|>1$ we have

$$
n^{1 / 2 \delta}\left(A|x|^{\delta}+B\right)^{1 / \delta}=n^{1 / 2 \delta}|x|\left(A+\frac{B}{|x|^{\delta}}\right)^{1 / \delta} \leqslant A_{1}|x| .
$$

In the case $|x| \leqslant 1$,

$$
n^{1 / 2 \delta}\left(A|x|^{\delta}+B\right) \leqslant A_{1}
$$

Therefore in both cases $|y|^{1 / \delta} \leqslant A_{1}(|x|+1)$. Since $2|x| \leqslant|x|^{2}+1$, we have $|y|^{2 / \delta} \leqslant A_{1}^{2}\left(|x|^{2}+2|x|+1\right) \leqslant 2 A_{1}^{2}\left(|x|^{2}+1\right)$. Similarly $|\bar{y}|^{1 / \delta} \leqslant A_{1}(|x|+1),|\bar{y}|^{2 / \delta}$ $\leqslant 2 A_{1}^{2}\left(|x|^{2}+1\right)$. By virtue of the above inequalities we receive

$$
\begin{gathered}
L_{1}|x|+L_{2}|y|^{1 / \delta}+L_{3}|\bar{y}|^{1 / \delta}+L_{4} \leqslant \bar{L}_{1}|x|+\bar{L}_{2}, \\
L_{\mathbf{6}}|x|^{2}+L_{\mathbf{6}}|y|^{2 / \delta}+L_{7}|\bar{y}|^{2 / \delta}+L_{\mathbf{8}} \leqslant \bar{L}_{3}|x|^{2}+\bar{L}_{4},
\end{gathered}
$$

$\bar{L}_{1}, \bar{L}_{2}, \bar{L}_{3}, \bar{L}_{4}$ being certain positive constants. It means that for functions $u_{i}^{(1)}, u_{i}^{(2)}$ of class $Z_{\delta}$ inequalities of form (3.4) are satisfied. This completes the proof. 


\section{§ 5. A certain theorem on the existence of a solution of} the (FI)-problem. As in paper [6], we will prove the following

Lемma. For $K>\frac{1}{2} \bar{m} \sqrt{\frac{n L_{3}+\lambda}{L_{0}}}$ and $k_{0}>0$, the inequality

$$
\frac{H(x, t ; K)}{H\left(x, t ; K+k_{0}\right)} \leqslant \exp \left(-k_{0}|x|^{2}\right)
$$

holds for $(x, t) \in \overline{D^{h_{0}}}$, where $h_{0}=(1-\gamma) / \mu\left(K+k_{0}\right), \quad 0<\gamma<1, \mu(K)$ is defined by (3.5), and $v(K)$ by (3.6).

Proof. By virtue of (3.6), $\nu\left(K+k_{0}\right)>v(K)$. From (3.5) it follows that for $K>\frac{1}{2 m} \sqrt{\frac{\bar{n} L_{2}+\lambda}{L_{0}}}, k_{0}>0$, we have $\mu\left(K+k_{0}\right)>\mu(K)$. Hence

$$
\begin{aligned}
& \frac{H(x, t ; K)}{H\left(x, t ; K+k_{0}\right)}=\exp \left\{\frac{K \mid x^{2}}{1-\mu(\bar{K}) t}-\frac{K|x|^{2}}{1-\mu\left(K+k_{0}\right) t}-\frac{k_{0}|x|^{2}}{1-\mu\left(K+k_{0}\right) t}+\right. \\
& \left.\quad+\left[v(K)-v\left(K+k_{0}\right)\right] t\right\} \leqslant \exp \left\{-\frac{k_{0}|x|^{2}}{1-\mu\left(K+k_{0}\right) t}\right\} \leqslant \exp \left(-k_{0}|x|^{2}\right) .
\end{aligned}
$$

The lemma is thus proved.

Let us put $\Omega_{R}=\Sigma_{R}+C_{R}, \Omega_{R}^{h}=\Sigma_{R}^{h}+C_{R}^{h}$.

Hypothesis $(\mathrm{H})$. Let $\Phi_{i}(x, t) \quad(i=1, \ldots, n)$ be arbitrary continuous functions belonging to elass $E_{2}$ in the domain $\bar{D}$. For every $R$ there exists a parabolic solution $u_{i}(x, t)(i=1, \ldots, n)$ of (1.1) regular in $D_{R}$ and such that $u_{i}(x, t)=\Phi_{i}(x, t)(i=1, \ldots, n)$ in the set $\Omega_{R}$.

We write $f_{s}(x, t)=F_{s}(x, t, 0,0, \ldots, 0)$. We will prove

TheOREM III. If

$1^{0}$ the $(\mathrm{H})$-hypothesis is satisfied,

$2^{\circ}$ system (1.1) is parabolic with regard to all the solutions mentioned in hypothesis (H),

$3^{\mathrm{o}}$ the functions $f_{s}(x, t)(s=1, \ldots, n)$ belong to $E_{2}$,

$4^{0}$ the functions $F_{s}(s=1, \ldots, n)$ satisfy the (I)-condition,

$5^{\circ} \varphi_{i}(x, t)(i=1, \ldots, n)$ are arbitrary given functions, defined, continuous, and belonging to $E_{2}$ in $\Sigma$;

then

1. there exists a solution of the (FI)-problem in $D^{h_{0}}$ (see $\left.\S 2\right)$, where $h_{0}$ is sufficiently small (defined as in the lemma),

2. this solution also belongs to class $E_{2}$ in $D^{h_{0}}$.

Proof. Let us choose $n$ functions $\Phi_{i}(x, t)$ continuous and of class $E_{2}\left(M, K_{0}\right)$ in $\bar{D}$, so that $\Phi_{i}(x, t)=\varphi_{i}(x, t) \quad(i=1, \ldots, n)$ for $(x, t) \in \Sigma$. We may assume that all the functions $\varphi_{\imath}(x, t), \Phi_{i}(x, t)$ and $f_{\imath}(x, t)$ $(i=1, \ldots, n)$ belong to $E_{2}\left(M, K_{0}\right)$ with the same constants $M$ and $K_{0}$. 
Let $\left\{R_{a}\right\}$ be an increasing sequence, $R_{\alpha} \rightarrow \infty$ as $\alpha \rightarrow \infty$. Let us fix natural numbers $\alpha$ and $\beta, \beta>\alpha$. By $u_{i}^{\alpha}(x, t)(i=1, \ldots, n)$ we denote a solution of (1.1), determined and regular in $D_{R_{a}}$, which satisfies the condition

$$
u_{i}^{a}(x, t)=\Phi_{i}(x, t) \quad(i=1, \ldots, n) \quad \text { for } \quad(x, t) \in \Omega_{R_{a}} .
$$

According to the (H)-hypothesis such a solution exists.

Similarly we define $u_{i}^{\beta}(x, t)(i=1, \ldots, n)$ in $D_{R_{\beta}}$.

For the functions $u_{i}^{a}(x, t)(i=1, \ldots, n)$ we have in $D_{R_{a}}$

$$
\frac{\partial u_{s}^{a}}{\partial t}=F_{s}\left(x, t, u_{i}^{a}, \frac{\partial u_{s}^{a}}{\partial x_{j}}, \frac{\partial^{2} u_{s}^{a}}{\partial x_{j} \partial x_{k}}\right) \quad(s=1, \ldots, n) \text {. }
$$

Next, we introduce the functions $v_{i}^{\alpha}, v_{i}^{\beta}$ determined by the relations

$$
u_{i}^{a}=v_{i}^{a} H(x, t ; K), \quad u_{i}^{\beta}=v_{i}^{\beta} H(x, t ; K) \quad(i=1, \ldots, n),
$$

where $H(x, t ; K)$ is defined by $(3.2)$; at the same time we choose

$$
K>\max \left(K_{0}, \frac{1}{2 m} \sqrt{\frac{\bar{n} L_{3}+\lambda}{L_{0}}}\right) .
$$

The functions $v_{i}^{a}$ satisfy the system

$$
\begin{aligned}
& \frac{\partial v_{s}^{a}}{\partial t} H+v_{s}^{a} \frac{\partial H}{\partial t}= \\
& F_{s}\left(x, t, v_{i}^{a} H, \frac{\partial v_{s}^{a}}{\partial x_{j}} H+v_{s}^{a} \frac{\partial H}{\partial x_{j}}, \frac{\partial^{2} v_{s}^{a}}{\partial x_{j} \partial x_{k}} H+\frac{\partial v_{s}^{a}}{\partial x_{k}} \frac{\partial H}{\partial x_{j}}+\frac{\partial v_{s}^{a}}{\partial x_{j}} \frac{\partial H}{\partial x_{k}}+v_{s}^{a} \frac{\partial^{2} H}{\partial x_{j} \partial x_{k}}\right)- \\
& -F_{s}\left(x, t, v_{i}^{a} H, \frac{\partial v_{s}^{a}}{\partial x_{j}} H+v_{s}^{a} \frac{\partial H}{\partial x_{j}}, 0+\frac{\partial v_{s}^{a}}{\partial x_{k}} \frac{\partial H}{\partial x_{j}}+\frac{\partial v_{s}^{a}}{\partial x_{j}} \frac{\partial H}{\partial x_{k}}+v_{s}^{a} \frac{\partial^{2} H}{\partial x_{j} \partial x_{k}}\right)+ \\
& +F_{s}\left(x, t, v_{i}^{a} H, \frac{\partial v_{s}^{a}}{\partial x_{j}} H+v_{s}^{a} \frac{\partial H}{\partial x_{j}}, 0+\frac{\partial v_{s}^{a}}{\partial x_{k}} \frac{\partial H}{\partial x_{j}}+\frac{\partial v_{s}^{a}}{\partial x_{j}} \frac{\partial H}{\partial x_{k}}+v_{s}^{a} \frac{\partial^{2} H}{\partial x_{j} \partial x_{k}}\right)- \\
& \quad-F_{s}(x, t, 0,0, \ldots, 0)+F_{s}(x, t, 0,0, \ldots, 0) .
\end{aligned}
$$

We consider the part $D^{h}$ of $D$ contained in the strip $0<t<h$, where $h \leqslant T$ will be determined later. We retain the notation of the domains and their boundaries introduced in $\S 3$.

Since the functions $\Phi_{i}(x, t)$ belong to $E_{2}\left(M, K_{0}\right)$, in $\Omega_{R_{a}}^{h}$ each function $v_{i}^{a}$ satisfies the inequality $\left|v_{i}^{a}(x, t)\right| \leqslant M(i=1, \ldots, n)$. We put

$$
A_{a}=\max _{(i)} \sup _{(x, t) \in \overline{D_{R_{\alpha}}^{h}}}\left|v_{i}^{a}(x, t)\right|
$$

First of all we will show that, for every $\alpha,\left|v_{i}^{\alpha}(x, t)\right| \leqslant M$ in the domain $\overline{D_{R_{\alpha}}^{h}}$. There exist an index $i_{a}$ and a point $\left(x_{a}, t_{a}\right) \in \overline{D_{R_{a}}^{h}}=\left(D_{R_{a}}^{h}+S_{R_{a}}^{h}\right)+\Omega_{R_{a}}^{h}$ such 
that $A_{a}=\left|v_{i_{\alpha}}^{a}\left(x_{\alpha}, t_{a}\right)\right|$. If $\left(x_{\alpha}, t_{\alpha}\right) \in \Omega_{R_{a}}^{h}$, then our assertion follows from the preceding remark. Suppose, therefore, that $\left(x_{\alpha}, t_{a}\right) \in D_{R_{\alpha}}^{h}+S_{R_{a}}^{h}$. If $v_{i_{a}}^{a}\left(x_{a}, t_{a}\right) \geqslant 0$, then at the point $\left(x_{a}, t_{a}\right)$ we have

$$
\frac{\partial v_{i_{a}}^{a}}{\partial t} \geqslant 0, \quad \frac{\partial v_{i_{a}}^{a}}{\partial x_{j}}=0 \quad(j=1, \ldots, m) \quad \text { as well as } \quad \sum_{j, k=1}^{m} \frac{\partial^{2} v_{i_{a}}^{a}}{\partial x_{j} \partial x_{k}} \lambda_{j} \lambda_{k} \leqslant 0
$$

for any real vector $\left(\lambda_{1}, \ldots, \lambda_{m}\right)$. Substituting $x=x_{a}, t=t_{\alpha}$ in the equation with the index $i_{\alpha}$ of (5.4) and taking advantage of the definition of parabolic equations, we find that the difference of the first two terms in this equation is non-positive. The difference of the next two terms is estimated according to the (L)-condition and we obtain

$$
\begin{aligned}
& \frac{\partial v_{i_{\alpha}}^{\alpha}}{\partial t} \leqslant \frac{v_{i_{\alpha}}^{\alpha}}{H}\left[L_{0} \sum_{j, k=1}^{m}\left|\frac{\partial^{2} H}{\partial x_{j} \partial x_{k}}\right|+\left(L_{1}\left|x_{\mid}\right|+L_{2}\right) \sum_{j=1}^{m}\left|\frac{\partial H}{\partial x_{j}}\right|+\right. \\
& \left.+\left(L_{3}|x|^{2}+L_{4}\right) n H-\frac{\partial H}{\partial t}\right]+f_{i_{a}}^{(1)}\left(x_{a}, t_{a}\right),
\end{aligned}
$$

where $f_{i_{a}}^{(1)}(x, t)=\frac{1}{H} f_{i_{a}}(x, t)$ Since $f_{i_{a}}(x, t) \in E_{2}\left(M, K_{0}\right)$, it follows from (3.2) and (5.3) that $\left|f_{i_{a}}^{(1)}\left(x_{a}, t_{a}\right)\right| \leqslant M$.

The constants $\mu(K), \nu(K)$ in the function $H(x, t ; K)$ and the number $h(K)$ can be chosen-in the same way as in the proof of theorem Iin such a way that the expression between the brackets in (5.5) is smaller than $-1 \cdot H$. Therefore at $\left(x_{a}, t_{a}\right)$ we have

$$
0 \leqslant \frac{\partial v_{i_{a}}^{a}}{\partial t} \leqslant-v_{i_{a}}^{a}+M
$$

whence $v_{i_{a}}^{a}\left(x_{a}, t_{a}\right) \leqslant M$. If $v_{i_{a}}^{a}\left(x_{a}, t_{a}\right)<0$, then similarly it can be shown that $v_{i_{a}}^{\alpha}\left(x_{a}, t_{a}\right) \geqslant-M$, and hence, finally, $\left|v_{i}^{\alpha}(x, t)\right| \leqslant M \quad(i=1, \ldots, n)$ in the domain $\overline{D_{R_{a}}^{h}}$. M does not depend on $a$; thus we have also

We put

$$
\left|v_{i}^{\beta}(x, t)\right| \leqslant M \quad(i=1, \ldots, n) \quad \text { in } \overline{D_{R_{\beta}}^{h}} .
$$

$$
u_{i}^{\alpha \beta}=u_{i}^{\alpha}-u_{i}^{\beta}, \quad v_{i}^{\alpha \beta}=v_{i}^{\alpha}-v_{i}^{\beta}
$$

Consequently,

$$
u_{i}^{\alpha \beta}=v_{i}^{\alpha \beta} H(x, t ; K) \quad \text { and } \quad\left|v_{i}^{\alpha \beta}\right| \leqslant 2 M \quad \text { in the domain } \overline{D_{R_{a}}^{h}} .
$$

For the functions $u_{i}^{\alpha}, u_{i}^{\beta}, u_{i}^{a \beta}(i=1, \ldots, n)$ we receive the system of the equations

$$
\frac{\partial u_{s}^{a \beta}}{\partial t}=F_{s}\left(x, t, u_{i}^{a}, \frac{\partial u_{s}^{a}}{\partial x_{j}}, \frac{\partial^{2} u_{s}^{a}}{\partial x_{j} \partial x_{k}}\right)-F_{s}\left(x, t, u_{i}^{\beta}, \frac{\partial u_{s}^{\beta}}{\partial x_{j}}, \frac{\partial^{2} u_{s}^{\beta}}{\partial x_{j} \partial x_{k}}\right)
$$


Further, we introduce the functions $\stackrel{*}{*}_{i}^{\alpha}, \stackrel{*_{i}^{\beta}}{*}$ according to the relations

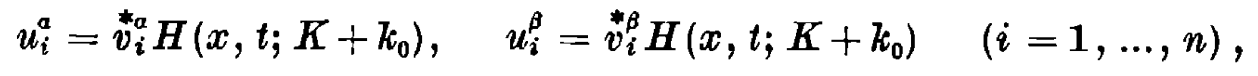

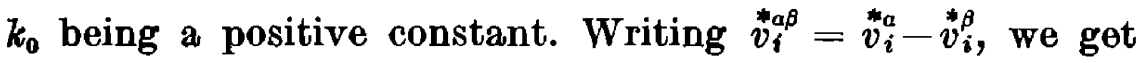

$$
u_{i}^{a \beta}={\stackrel{k}{*} \boldsymbol{k}_{i} \beta}_{H}\left(x, t ; K+k_{0}\right) \quad(i=1, \ldots, n) .
$$

In view of (5.8), (5.9) and (5.10) we obtain for the functions $\stackrel{*}{*}_{i}^{a}, \stackrel{*_{v}^{*}}{v_{i}}, \stackrel{*_{i} \beta}{v_{i}}$ the system

$$
\begin{aligned}
& \frac{\partial v_{s}^{* a \beta}}{\partial t} H+{\stackrel{*}{*}{ }_{s}^{a \beta}}^{\alpha} \frac{\partial H}{\partial t}= \\
& F_{s}\left(x, t, v_{i}^{*_{a}} H, \frac{\partial v_{s}^{*_{a}}}{\partial x_{j}} H+{\stackrel{v}{v_{s}}}_{s} \frac{\partial H}{\partial x_{j}}, \frac{\partial^{2} \boldsymbol{v}_{s}^{*_{a}}}{\partial x_{j} \partial x_{k}} H+\frac{\partial v_{s}^{*_{a}}}{\partial x_{k}} \frac{\partial H}{\partial x_{j}}+\frac{\partial v_{s}^{*_{a}}}{\partial x_{j}} \frac{\partial H}{\partial x_{k}}+v_{s}^{*_{a}} \frac{\partial^{2} H}{\partial x_{j} \partial x_{k}}\right)-
\end{aligned}
$$

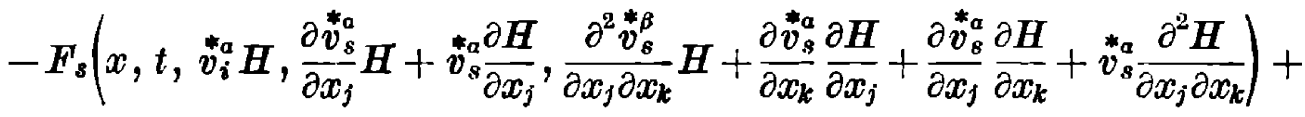

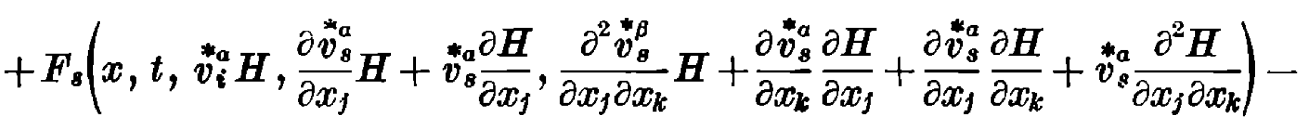

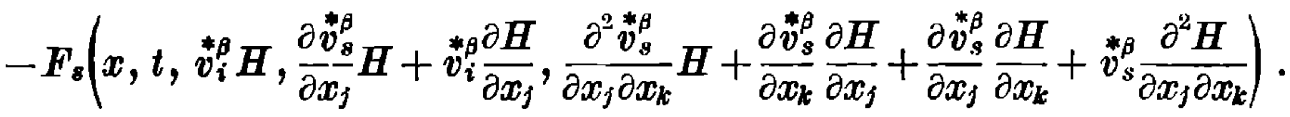

Let us write

$$
A_{\mathrm{\alpha} \beta}=\max _{(i)} \sup _{(x, t) \in \overline{D_{R_{a}}^{h_{0}}}}\left|v_{i}^{k_{\alpha} \beta}(x, t)\right|,
$$

where $h_{0}, 0<h_{0}<h$, will be determined later. There exist an index $i_{\alpha \beta}$ and a point $\left(x_{a \beta}, t_{a \beta}\right) \in \overline{D_{R_{a}}^{h_{0}}}=\left(D_{R_{\alpha}}^{h_{0}}+S_{R_{a}}^{h_{0}}\right)+\Sigma_{R_{a}}^{h_{0}}+C_{R_{a}}^{h_{0}}$ such that $A_{a \beta}$ $=\left|\boldsymbol{v}_{i_{\alpha \beta} \beta}^{* a \beta}\left(x_{\alpha \beta}, t_{a \beta}\right)\right|$. By an argument similar to that in the proof of theorem I, we show that, for a convenient choice of $\mu(K), \nu(K)$ and $h_{0}$, if $\left(x_{a \beta}, t_{a \beta}\right) \in D_{R_{a}}^{h_{0}}+S_{R_{a}}^{h_{0}}$, then $\dot{v}_{i_{a \beta}}^{*}\left(x_{a \beta}, t_{\alpha \beta}\right)=0$. If $\left(x_{\alpha \beta}, t_{\alpha \beta}\right) \in \Sigma_{R_{a}}^{h_{0}}$, then by the definition of $u_{i}^{a}$ we have also $v_{i_{a \beta}}^{*_{a \beta}}\left(x_{a \beta}, t_{a \beta}\right)=0$. If finally $\left(x_{a \beta}, t_{a \beta}\right) \in C_{R_{a}}^{h_{0}}$, then by (5.7), (5.10) and by the lemma we have at $\left(x_{a \beta}, t_{a \beta}\right)$

$$
\left|\stackrel{*}{v} a \beta_{i}(x, t)\right| \leqslant \frac{2 M H(x, t ; K)}{H\left(x, t ; K+k_{0}\right)} \leqslant 2 M \exp \left(-k_{0}|x|^{2}\right) \quad(i=1, \ldots, n) .
$$

Thus we have proved that the inequality

$$
\left|\hat{v}_{i}^{* \beta \beta}(x, t)\right| \leqslant 2 M \exp \left(-k_{0} R_{a}^{2}\right) \quad(i=1, \ldots, n)
$$

holds true in $\overline{D_{R_{a}}^{h_{0}}}$. 
Now let $r$ be an arbitrary positive number and let us consider the domain $D_{r}^{h_{0}}$. We choose $\alpha$ so that $R_{a}>r$. By virtue of (5.10) and (5.12) we find

$$
\left|u_{i}^{\alpha \beta}(x, t)\right| \leqslant 2 M N_{0} \exp \left(-k_{0} R_{a}^{2}\right) \quad(i=1, \ldots, n) \quad \text { for } \quad(x, t) \in \overline{D_{r}^{h_{0}}},
$$

where $N_{0}=\frac{\max }{\overline{D_{r}^{h_{0}}}} H\left(x, t ; K+k_{0}\right)$. Hence it follows that for every $\varepsilon>0$ there exists an index $a_{0}(\varepsilon), R_{a_{0}}>r$, such that for $\beta>a>a_{0}$ the inequalities

$$
\left|u_{i}^{a}-u_{i}^{\beta}\right|<\varepsilon \quad(i=1, \ldots, n)
$$

hold for $(x, t) \in \overline{D_{r}^{h_{0}}}$. It means that the sequences

$$
\left\{u_{1}^{a}(x, t\},\left\{u_{2}^{a}(x, t)\right\}, \ldots,\left\{u_{n}^{a}(x, t)\right\}\right.
$$

are almost uniformly convergent in $\overline{D^{h_{0}}}$ as $a \rightarrow \infty$. Observe that the limit functions $U_{1}(x, t), U_{2}(x, t), \ldots, U_{n}(x, t)$ of these sequences belong to class $E_{2}$. In fact,

$$
U_{i}=\lim _{a \rightarrow \infty} u_{i}^{a}=\lim _{a \rightarrow \infty} v_{i}^{a} H(x, t ; K) \quad(i=1, \ldots, n)
$$

and $\left|v_{i}^{\alpha}\right| \leqslant M$ for every $i$ and $a$, whence

$$
\left|U_{i}(x, t)\right| \leqslant M \exp \left\{\frac{K|x|^{2}}{1-\mu t}+v t\right\} \quad(i=1, \ldots, n), \quad \text { q.e.d. }
$$

Now we will show that the functions $U_{i}(x, t)(i=1, \ldots, n)$ constitute the solution of the (FI)-problem in $D^{h_{0}}$. Obviously

$$
U_{i}(x, t)=\varphi_{i}(x, t) \quad(i=1, \ldots, n) \quad \text { for } \quad(x, t) \in \Sigma^{h_{0}} .
$$

It remains to prove that $U_{i}(x, t)(i=1, \ldots, n)$ is a (parabolic) solution of (1.1). It is sufficient to prove this in the domain $D_{r}^{h_{0}}$ for an arbitrary $r$. Let $u_{i}^{r}(x, t)(i=1, \ldots, n)$ be a parabolic solution of (1.1), regular in $D_{r}^{h_{0}}$, satisfying the condition $u_{i}^{r}(x, t)=\varphi_{\imath}(x, t)(i=1, \ldots, n)$ for $(x, t)$ $\epsilon \Sigma_{\tau}^{h_{0}}$ and identical with $U_{i}(x, t)$ for $(x, t) \epsilon C_{r}^{h_{0}}$. From the almost uniform convergence of sequences (5.13) it follows that for every $\varepsilon>0$ there is an index $a_{1}(\varepsilon), R_{a_{1}}>r$, such that for $a>a_{1}$ the inequalities

$$
\left|U_{i}-u_{i}^{a}\right|<\frac{\varepsilon}{N_{1}+1} \quad(i=1, \ldots, n),
$$

where $N_{1}=\underset{D_{r}^{h_{0}}}{\max } H\left(x, t, K+k_{0}\right)$, hold for $(x, t) \in \overline{D_{r}^{h_{0}}}$. In the set $\Omega_{r}^{h_{0}}$ we have $U_{i}=u_{i}^{r} ;$ therefore

$$
\left|u_{i}^{r}-u_{i}^{a}\right|<\frac{\varepsilon}{N_{1}+1} \quad(i=1, \ldots, n) \quad \text { for } \quad a>\alpha_{1}, \quad(x, t) \in \Omega_{r}^{h_{0}} .
$$


The functions

$$
\begin{aligned}
& \stackrel{*}{v}_{i}^{r}=\frac{u_{i}^{r}}{H\left(x, t ; \bar{K}+k_{0}\right)}, \quad \stackrel{*_{a}}{v_{i}}=\frac{u_{i}^{\alpha}}{H\left(x, t ; K+k_{0}\right)}, \quad \stackrel{*_{v}^{r a}}{r_{i}}=\stackrel{*_{r}^{*}}{v_{i}}-\stackrel{*}{* a}_{i} \\
& (i=1, \ldots, n)
\end{aligned}
$$

fulfil a system similar to (5.11). Let us put

$$
A_{r \alpha}=\max _{(i)} \sup _{(x, l) \in \overline{D_{r}^{h_{0}}}}\left|\hat{v}_{\dot{i}}^{r a}(x, t)\right|
$$

There exist an index $i_{r a}$ and a point $\left(x_{r \alpha}, t_{r \alpha}\right) \in \overline{D_{r}^{h_{0}}}=\left(D_{r}^{h_{0}}+S_{r}^{h_{0}}\right)+\Omega_{r}^{h_{\theta}}$ such that $A_{r a}=\left|v_{i_{r a}}^{r_{r}}\left(x_{r_{a}}, t_{r a}\right)\right|$. Reasoning as before, we find that if $\left(x_{r a}, t_{r a}\right) \in D_{r}^{h_{a}}+S_{r}^{h_{0}}$, then $v_{i_{r a}}^{*_{r a}}\left(x_{r a}, t_{r a}\right)=0$. If $\left(x_{r a}, t_{r a}\right) \in \Omega_{r}^{h_{0}}$, then from (5.15) and (5.16) it follows that

Consequently,

$$
\left|v_{i_{r \beta}}^{*_{r a}}\left(x_{r a}, t_{r a}\right)\right|<\frac{\varepsilon}{N_{1}+1}
$$

$$
\left|v_{i}^{* r a}(x, t)\right|<\frac{\varepsilon}{N_{1}+1} \quad(i=1, \ldots, n)
$$

everywhere in $\overline{D_{r}^{h_{0}}}$. Taking into consideration (5.16), we have

$$
\left|u_{i}^{r}-u_{i}^{a}\right|<\frac{N_{1} \varepsilon}{N_{1}+1}
$$

Hence and from (5.14) we receive the inequalities

$$
\left|U_{i}-u_{i}^{r}\right| \leqslant\left|U_{i}-u_{i}^{a}\right|+\left|u_{i}^{a}-u_{i}^{r}\right|<\varepsilon \quad(i=1, \ldots, n) \quad \text { for } \quad(x, t) \in \overline{D_{r}^{h_{0}}},
$$

which means that $U_{i}(x, t) \equiv u_{i}^{r}(x, t) \quad(i=1, \ldots, n)$ in the domain $\overline{D_{r}^{s_{0}}}$. Therefore $U_{i}(x, t)(i=1, \ldots, n)$ is the solution of the (FI)-problem in $D^{h_{0}}$. As we have proved before, this solution belongs to class $E_{2}$.

According to theorem $\mathrm{I}$ the functions $U_{i}(x, t)(i=1, \ldots, n)$ constitute the unique solution of the problem in question.

If the domain $D$ is the whole strip

$$
D\left\{-\infty<x_{i}<+\infty(i=1, \ldots, m), 0<t<T\right\},
$$

then theorems I-III remain true for the Cauchy problem, i.e. the problem of the existence and uniqueness of the solution $u_{i}(x, t)(i=1, \ldots, n)$ of system (1.1), satisfying the initial condition

$$
u_{i}(x, 0)=\varphi_{i}(x) \quad(i=1, \ldots, n),
$$

where the initial data $\varphi_{i}(x)$ are continuous functions, defined and belonging to class $E_{2}$ on the hyperplane $t=0$. 


\section{References}

[1] P. Besala, On solutions of non-linear parabolic equations defined in unbounded domains, Bull. Acad. Polon. Sci., Sér. sci. math., astr. et phys. 9 (1961), p. 531-535.

[2] С. Д. Эйдельман, $O$ задаче Коши дял нелинейных и квази-линейных параболических систем, Д.А.Н. С.С.С.Р., 116 (1957), p. 930-932.

[3] A. Friedman, On the uniqueness of the Cauchy problem for parabolic equations, Amer. Journ. Math. 81.2 (1959), p. 503-511.

[4] E. Holmgren, Sur les solutions quasi-analytiques de léquation de la chaleur, Arkiv för Matematik, Astronomi och Fysik 18 (1924), p. 1-5.

[5] M. Krz yżański, Sur les solutions des équations du type parabolique délerminées dans une région illimitée, Bull. Amer. Math. Soc. 47 (1941), p. $911-915$.

[6] - Sur les solutions de l'équation linéaire du type parabolique déter minées par. les conditions initiales, Ann. Soc. P’olon. Math. 18 (1945), p. 145-156.

[7] - - Note complémentaire, Ann. Soc. Polon. Math. 20 (1947), p. 7-9.

[8] - Évaluations des solutions de l'équation aux dérivées partielles du type parabolique, déterminées dans un domaine non borné, Ann. Polon. Math. 4 (1957), p. 93-97.

[9] - Certaines inégalités relatives aux solutions de l'équation parabolique linéaire normale, Bull. Acad. Polon. Sci., Série de sci. math., astr. et phys. 7 (1959), p. 131-135.

[10] - Równania różniczkowe czqstkowe rzędu drugiego, cz. I, Warszawa 1957.

[11] H. Milicer-Grużewska, Le théorème d'unicité de la solution du système parabolique des équations linéaires avec les coefficients höldériens, Bull. Acad. Polon. Sci., Série de sci. math., astr. et phys. 7 (1959), p. 593-599.

[12] - Le second théorème d'unicité de solution d'un système parabolique d'équations linéaires avec les coefficients höldériens, Bull. Acad. Polon. Sci. Série de sci. math., astr. et phys. 7 (1959), p. 719.720.

[13] W. Mlak, Differential inequalities of parabolic type, Ann. Polon. Math. 3 (1957), p. 349-354.

[14] - The first boundary-value problem for a non-linear parabolic equation, Ann. Polon. Math. 5 (1958-1959), p. 257.262.

[15] M. Picone, Sul problema della propagazione del calore in un mezzo privo di frontiera, Math. Ann. 10 (1929), p. 701-712.

[16] W. Pogorzelski, Propriétés des intégrales généralisées de Poigson-Weierstrass et problème de Cauchy pour un système parabolique, Ann. Ec. Norm. 76 (1959), p. $125-149$.

[17] J. Szarski, Sur la limitation et l'unicité des solutions d'un système non-linéaire d'équations paraboliques aux dérivées partielles du second ordre, Ann. Polon. Math. 2 (1955), p. 237-249.

[18] — Sur la limilation et l'unicité des solutions des problèmes de Fourier pour un système non linéaive d'équations paraboliques, Ann. Polon. Math. 4 (1959), p. 211-216.

[19] Т. Д. Вентцель, Первая краевая задача и задача Коши для көазилинейного параболического урдвнения со мнггими пространственными переменныли, Математический сборник (новая серия), 41.4 (1957), р. 499-520.

[20] Я. И. Житомирский, Задзиа Коии для параболических систем лмнейных удавнений ө частных произяодных с растуцики коэффичиентами, Известия Высших Учебньх Заведений, Мэтематика 1 (1959), р. 55-74. 